\title{
LETTER
}

Chronic lymphocytic leukemia

\section{Short term results of vaccination with adjuvanted recombinant varicella zoster glycoprotein E during initial BTK inhibitor therapy for CLL or lymphoplasmacytic lymphoma}

\author{
Clive S. Zent $\mathbb{C}^{1} \cdot$ Michael T. Brady ${ }^{1} \cdot$ Carolyne Delage $^{1} \cdot$ Myla Strawderman $^{2} \cdot$ Nathan Laniewski $\mathbb{1}^{3}$.

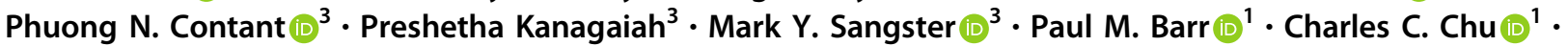 \\ David J. Topham (iD ${ }^{3} \cdot$ Jonathan W. Friedberg ${ }^{1}$
}

Received: 16 May 2020 / Revised: 25 September 2020 / Accepted: 19 October 2020 / Published online: 30 October 2020

(c) The Author(s), under exclusive licence to Springer Nature Limited 2020

\section{To the Editor:}

Reactivation of latent infection by varicella zoster (VZR) usually causes shingles with less common complications including postherpetic neuralgia, retinitis, cranial nerve palsies, myelitis, meningitis, cerebrovascular events, pancreatitis, hepatitis, and disseminated varicella. Although VZR usually responds to therapy, complications can be fatal or cause long term disability. Risk of VZR increases with age and other causes of immunosuppression [1].

Patients with hematological malignancies have a significantly increased risk of VZR [2, 3]. In the setting of chronic lymphocytic leukemia/small lymphocytic lymphoma (CLL) and lymphoplasmacytic lymphoma/Waldenstrom macroglobulinemia (LPL), patients have early onset and often severe immunocompromise that increases the risk of infections including VZR [4-6]. Unfortunately the attenuated live $\mathrm{VZ}$ vaccine is contraindicated in this population

Supplementary information The online version of this article (https:// doi.org/10.1038/s41375-020-01074-4) contains supplementary material, which is available to authorized users.

Clive S. Zent

clive_zent@urmc.rochester.edu

1 Wilmot Cancer Institute and Department of Medicine, University of Rochester Medical Center, 601 Elmwood Avenue, Rochester, NY 14642, USA

2 Department of Biostatistics and Computational Biology, University of Rochester Medical Center, 601 Elmwood Avenue, Rochester, NY 14642, USA

3 David H. Smith Center for Vaccine Biology and Immunology, Department of Microbiology and Immunology, University of Rochester Medical Center, 601 Elmwood Avenue, Rochester, NY 14642, USA because of risk of infection related complications [7]. In contrast, the newer recombinant $\mathrm{VZ}$ glycoprotein $\mathrm{E}$ (rVZgE) vaccine utilizing a highly effective adjuvant system is safer and potentially more effective [2, 8, 9]. However, data from the phase $3 \mathrm{rVZgE}$ vaccine trial in patients with hematological malignancies have limited applicability to current management of CLL because of exclusion of patients treated with oral targeted therapies [2].

Inhibitors of Bruton tyrosine kinase (BTKi) have improved the efficacy and decreased the toxicity of CLL [10, 11] and LPL [12] therapy. Unlike immunosuppressive chemoimmunotherapy, BTKi minimally alter immune function [13], or short term risk of infection in CLL patients [6]. CLL patients on ibrutinib respond sub-optimally to influenza vaccine [14] but it is uncertain if this is a de novo or anamnestic immune response [13]. We are not aware of any published data on the immunological response to rVZgE vaccine in patients with CLL or other indolent Bcell lymphomas on concurrent BTKi therapy.

This study tested the hypothesis that $\mathrm{rVZgE}$ vaccination of CLL or LPL patients on initial BTKi therapy for progressive disease would result in humoral and cellular responses comparable to those previously reported for patients with other hematological diseases [2]. The study enrolled patients with CLL or LPL age $\geq 50$ years on treatment with a BTKi for $\geq 3$ months. The only other permitted therapy was rituximab which had to be completed $>1$ year prevaccination. Exclusions were VZR within 1 year, other proximal vaccination, or prevaccination lymphocyte count $>20 \times 10^{9} / \mathrm{L}$ to avoid interference with cellular assays. Study participants received two doses of $\mathrm{rVZgE}$ vaccine (Shingrix, GlaxoSmithKline, Brentford, United Kingdom) 8-12 weeks apart per product label with blood sampled prevaccination and $4 \pm 1$ weeks after the second vaccination. Control healthy donor blood specimens were from an 
approved protocol at the University of Rochester. Humoral vaccine response analysis compared concentrations of serum IgG specific for $\mathrm{VZgE}$ to selected non-VZ proteins measured by ELISA as previously described [15]. Cellular response analysis measured VZgE-specific T-cell responses in blood with $\geq$ twofold increases in the frequency of activated CD4+ T-cell clusters compared to prevaccination frequencies considered evidence of response. Evaluation of clinical response to vaccination was limited to selected attributable grade $>2$ adverse effects and VZR was not monitored. Supplementary data provide detail of methods and statistical analysis.

Thirty-two $(C L L=22, L P L=10)$ patients were vaccinated between January and September 2019 (Supplemental Table 2) with no vaccine attributable grade $>2$ adverse events. Twelve CLL patients had previous rituximab on clinical trials as previously described [10, 11]. Thirty patients had detectable serum VZgE-specific IgG prevaccination and all had detectable levels postvaccination (Fig. 1). Serum IgG specific for TTd and the H1 hemagglutinin of influenza was minimally changed after vaccination. The $\mathrm{VZgE}$-specific serum IgG concentration geometric mean $(90 \% \mathrm{CI})$ increased from $1.7 \mu \mathrm{g} / \mathrm{mL}(1.2$, 2.3) prevaccination by 13.2 -fold $(8.0,21.6)$ to $22.5 \mu \mathrm{g} / \mathrm{mL}$

A

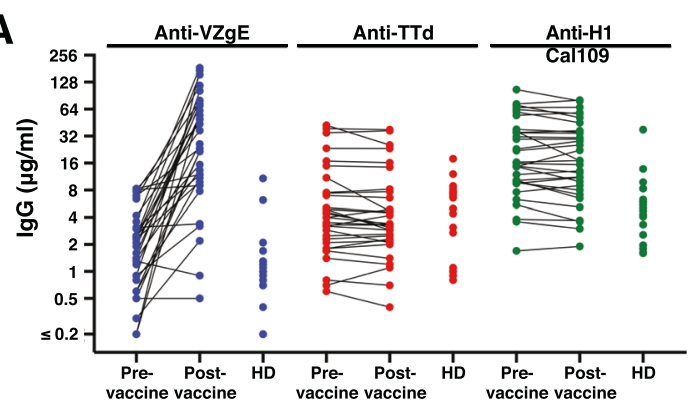

B

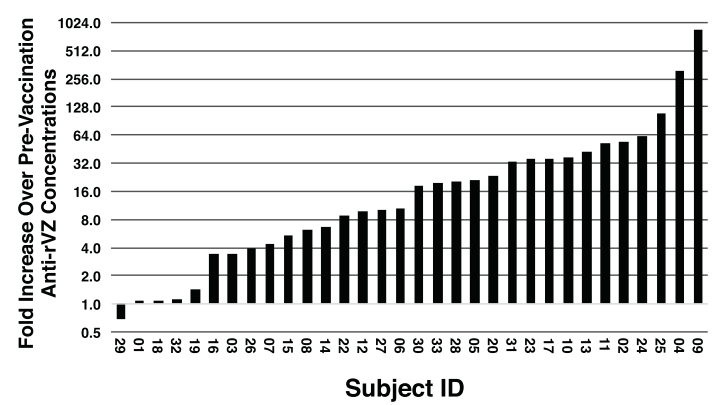

Fig. 1 Humoral response to vaccination. A Antigen-specific serum $\mathrm{IgG}$ concentrations $(\mu \mathrm{g} / \mathrm{mL}, \log$ scale) for varicella zoster glycoprotein $\mathrm{E}$ (anti-VZgE), tetanus toxoid (anti-TTd), and hemagglutinin of influenza virus A/California/4/2009 (H1N1) (anti-H1 Cal109), measured before and $\sim 4$ weeks after second vaccination with adjuvanted recombinant varicella zoster glycoprotein $\mathrm{E}$ vaccine. $\mathrm{HD}$ is serum $\mathrm{IgG}$ concentration in unvaccinated healthy donor controls. B Plot of fold change (log scale) in anti-VZgE in each patient after vaccination.
(14.4, 35.1) postvaccination. Twenty-four $(75 \%, 90 \% \mathrm{CI}$ $59.4,86.9)$ of 32 patients had a humoral response to vaccination using $\geq$ fourfold increase in concentration as evidence of seroconversion [9].

Cellular immune response to vaccination was determined by change in frequency of activated CD4 + T-cell clusters after stimulation with the VZgE peptide pool (Fig. 2). Highdimensional clustering analysis using FlowSOM identified six unique CD4+ clusters significantly associated with rVZgE vaccination (Supplementary Fig. 1). The sum of these clusters was used to determine cellular response. The prevaccination median (IQR) VZ-specific CD4+ T-cells of $0.0066 \%(0.0066,0.0171)$ increased 4.1 -fold $(2.4,6.8)$ to $0.0462 \%(0.0238,0.0731)$ after vaccination and $25(78 \%$, IQR $62.8,89.2$ ) of 32 patients had the $\geq$ twofold increase defining a cellular response to vaccination [9].

Of the $24(75 \%)$ subjects with a humoral immune response, $21(87.5 \%)$ also achieved a T-cell response (Supplementary Fig. 2A). For the eight subjects without a humoral immune response, only four $(50 \%)$ had a T-cell response. Four patients did not meet criteria for either a humoral or T-cell response. A significant association was found between humoral and T-cell responses with Fisher's exact test $(p=0.047)$. The Spearman Rank correlation was used to assess the strength and direction of the association between $\mathrm{T}$-cell and humoral changes. The correlation between humoral and T-cell fold increase was $0.22(90 \%$ $\mathrm{CI}=-0.09,0.48)$ and for the CLL subset was $0.36(90 \%$ $\mathrm{CI}=0.0,0.64)$. Exploratory analysis of associations between humoral vaccine responses and patient data (Supplementary Table 3) showed only a significant association between longer duration of prevaccination BTKi treatment ( $\geq$ median of 33 months) and a lower VZ-specific IgG serum concentration response (geometric mean increase 6.8 vs. 25.5 -fold, $p=0.02 ; \%$ response 56.3 vs. $93.8, p=0.04$ ) (Supplementary Fig. 2B).

This prospective study shows that patients with CLL or LPL on BTKi therapy can respond to $\mathrm{rVZgE}$ vaccine while on BTKi therapy. In the pivotal healthy subject clinical trial $\mathrm{rVZgE}$ vaccine decreased the rate of VZR by $97 \%$ [8] with humoral immune responses in $98 \%$ and T-cell responses in $93 \%$ of patients [9]. In contrast, patients with hematological malignancies had lower humoral $(65 \%)$ and T-cell $(84 \%)$ responses to vaccination [2]. We now show shows that CLL and LPL patients on treatment with BTKi achieve comparable rates of objective responses to $\mathrm{rVZgE}$ vaccine, providing the rationale for further evaluation of the efficacy of $\mathrm{rVZgE}$ vaccination in patients with indolent B-cell malignancies.

The criteria for determining immune response in this study are similar to those used previously $[2,9]$. These results are useful for assessing vaccine responses in a population of patients, but could be less informative about 

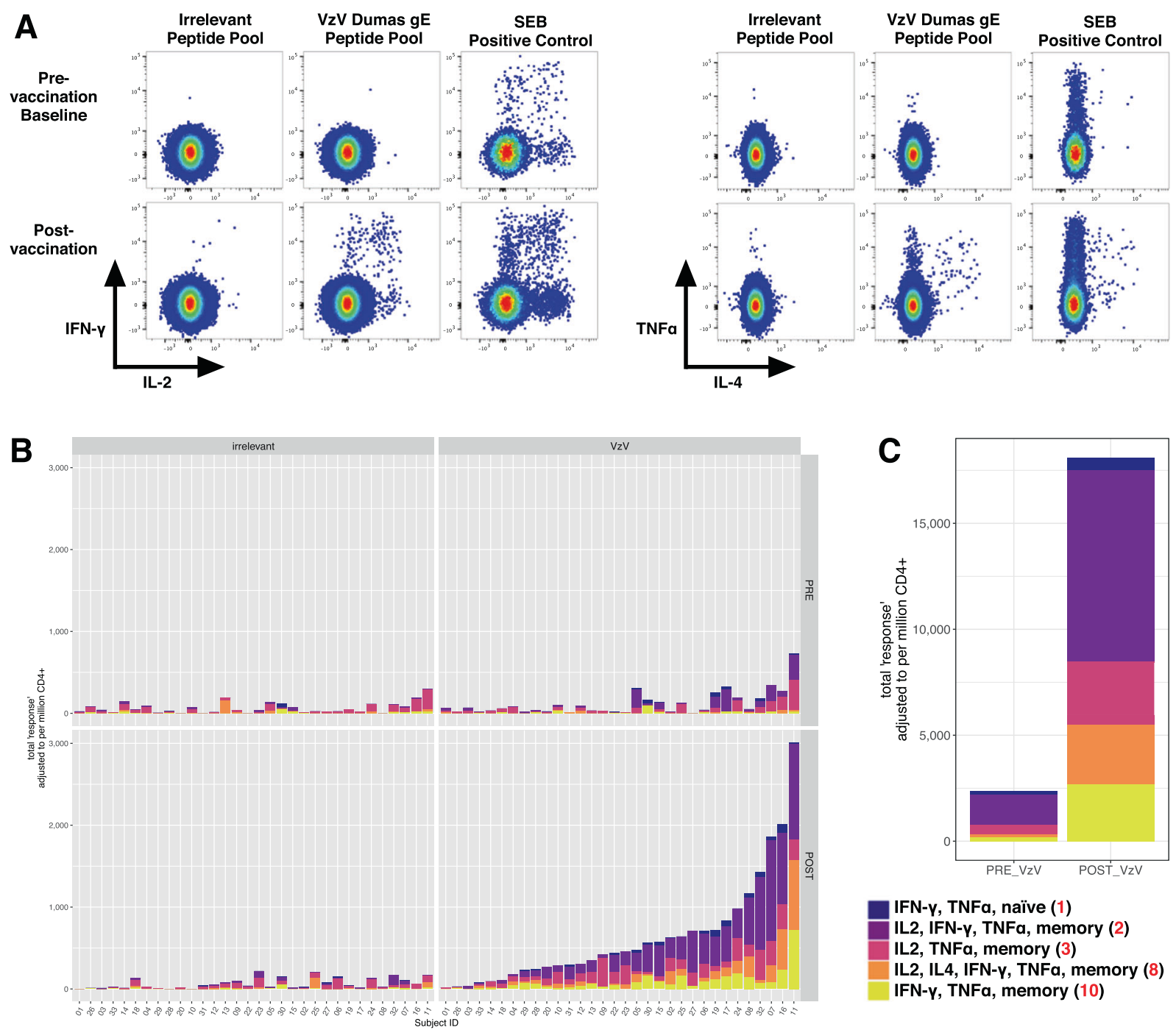

IFN-y, TNFa, naïve (1)

IL2, IFN-y, TNFa, memory (2)

IL2, TNFa, memory (3)

IL2, IL4, IFN-y, TNFa, memory (8)

IFN-y, TNFa, memory (10)

Fig. $2 \mathrm{CD} 4+\mathrm{T}$-cell response to $\mathrm{rVZgE}$ vaccination. A Representative flow cytometry pseudocolor plots showing specific cytokine producing CD4+ T-cells following in vitro stimulation. B Cell count (adjusted to per million $\mathrm{CD} 4+$ ) for the five FlowSOM clusters (lower right text legend) that represent a vaccine response, shown as a stacked bar for each individual subject $(n=32)$; subjects are rank-ordered according to total response in the postvaccination $\mathrm{VZgE}$ (' $\mathrm{VzV}$ ') group. C Cumulative sum of the response clusters, shown as a stacked barall subjects combined, pre- vs. postvaccination (respective irrelevant peptide background subtracted). individual patient responses to vaccination as detailed in the Supplementary data. An improved ability to predict individual immunity to VZR following vaccination will require additional studies to identify accurate biomarkers of protective immune responses.

Using published definitions to dichotomize responses, we showed a significant correlation between humoral and T-cell responses (Supplementary Fig. 2A) with 4 patients having no response to vaccine and 21 having both humoral and T-cell responses. In contrast when considered as continuous variables, Spearman Rank analysis did not show a significant correlation between humoral and T-cell responses (see Supplementary data). Our data are thus suggestive but not definitive of a correlation between humoral and T-cell immune response to vaccination.

Univariate testing for associations with immune response to vaccine focused on humoral immunity because of the availability of quantitative data, documented humoral immunity defects in CLL and LPL, and concerns that BTKi and prior rituximab therapy could inhibit humoral responses. The only significant correlation was between longer duration of BTKi therapy and poorer response to vaccination (Supplementary Table 3, Supplementary Fig. 2B) which suggests that prolonged BTKi therapy could inhibit humoral response as discussed further in the Supplementary data. Multivariate analysis was not performed because of the small size of 
the study population and the inclusion of two discrete lymphoid malignancies.

The strengths of this study are that it was a prospective clinical trial enrolling patients receiving BTKi as initial therapy with standard administration of vaccine and collection of samples which were analyzed in a specialized vaccine research laboratory. Limitations include the small number of subjects and the inclusion of two similar B-cell malignancies in this pilot study which was of limited scope given the concern that disease related immunosuppression and immune effects of BTKi would prevent any vaccinal effect. The durability of immune responses has not yet been documented because of the coronavirus pandemic. Only four patients had no response to vaccination limiting our ability to evaluated reasons for this failure. All these patients had CLL treated initially with six cycles of rituximab in combination with ibrutinib and had a longer (40-56 vs. median 33 months) duration of BTKi prevaccination.

In conclusion we report an unexpected high rate of $\operatorname{IgG}$ and $\mathrm{T}$-cell responses to $\mathrm{rVZgE}$ vaccination in patients with CLL or LPL on BTKi therapy. Ongoing and future trials will be needed to determine the clinical utility of vaccination in patients with indolent B-cell malignancies. If our results are validated, $\mathrm{rVZgE}$ vaccination should become part of the standard of care for patients with CLL or LPL on BTKi therapy.

Acknowledgements In memory of Carolyne Delage MD (7/1/1987-6/ 23/2018). We thank patients, Lynn Rich NP, Sharon Lewinski RN, Maureen Tremaine RN, Tania Orzol NP, Karl VanDerMeid, Delaney Dretto, Michael Flannery, and Cristin Sciortino for making this study possible. The study was supported by the Cadregari Endowment Fund of the Wilmot Cancer Institute, Wilmot Biostatistics and Bioinformatics Shared Resource, Research \& Academic IT, Clinical and Translational Science Institute grant (Grant \#UL1TR002001).

\section{Compliance with ethical standards}

Conflict of interest The authors declare that they have no conflict of interests.

Publisher's note Springer Nature remains neutral with regard to jurisdictional claims in published maps and institutional affiliations.

\section{References}

1. Kawai K, Gebremeskel BG, Acosta CJ. Systematic review of incidence and complications of herpes zoster: towards a global perspective. BMJ Open. 2014;4:e004833.

2. Dagnew AF, Ilhan O, Lee WS, Woszczyk D, Kwak JY, Bowcock $S$, et al. Immunogenicity and safety of the adjuvanted recombinant zoster vaccine in adults with haematological malignancies: a phase 3, randomised, clinical trial and post-hoc efficacy analysis. Lancet Infect Dis. 2019;19:988-1000.

3. Hansson E, Forbes HJ, Langan SM, Smeeth L, Bhaskaran K. Herpes zoster risk after 21 specific cancers: population-based case-control study. Br J Cancer. 2017;116:1643-51.

4. Forconi F, Moss P. Perturbation of the normal immune system in patients with CLL. Blood. 2015;126:573-81.

5. Hunter ZR, Manning RJ, Hanzis C, Ciccarelli BT, Ioakimidis L, Patterson CJ, et al. IgA and IgG hypogammaglobulinemia in Waldenstrom's macroglobulinemia. Haematologica. 2010;95:470-5.

6. Williams AM, Baran AM, Meacham PJ, Feldman MM, Valencia HE, Newsom-Stewart C, et al. Analysis of the risk of infection in patients with chronic lymphocytic leukemia in the era of novel therapies. Leuk Lymphoma. 2018;59:625-32.

7. Willis ED, Woodward M, Brown E, Popmihajlov Z, Saddier P, Annunziato PW, et al. Herpes zoster vaccine live: a 10 year review of post-marketing safety experience. Vaccine. 2017;35:7231-9.

8. Lal H, Cunningham AL, Godeaux O, Chlibek R, Diez-Domingo J, Hwang SJ, et al. Efficacy of an adjuvanted herpes zoster subunit vaccine in older adults. N Engl J Med. 2015;372:2087-96.

9. Cunningham AL, Heineman TC, Lal H, Godeaux O, Chlibek R, Hwang SJ, et al. Immune responses to a recombinant glycoprotein E herpes zoster vaccine in adults aged 50 years or older. J Infect Dis. 2018;217:1750-60.

10. Woyach JA, Ruppert AS, Heerema NA, Zhao W, Booth AM, Ding $\mathrm{W}$, et al. Ibrutinib regimens versus chemoimmunotherapy in older patients with untreated CLL. N Engl J Med. 2018;379:2517-28.

11. Shanafelt TD, Wang XV, Kay NE, Hanson CA, O'Brien S, Barrientos $\mathrm{J}$, et al. Ibrutinib-rituximab or chemoimmunotherapy for chronic lymphocytic leukemia. N Engl J Med. 2019;381:432-43.

12. Treon SP, Tripsas CK, Meid K, Warren D, Varma G, Green R, et al. Ibrutinib in previously treated Waldenstrom's macroglobulinemia. N Engl J Med. 2015;372:1430-40.

13. Pleyer C, Wiestner A, Sun C. Immunological changes with kinase inhibitor therapy for chronic lymphocytic leukemia. Leuk Lymphoma. 2018;59:2792-800.

14. Sun C, Gao J, Couzens L, Tian X, Farooqui MZ, Eichelberger $M C$, et al. Seasonal influenza vaccination in patients with chronic lymphocytic leukemia treated with ibrutinib. JAMA Oncol. 2016;2:1656-7.

15. Tesini BL, Kanagaiah P, Wang J, Hahn M, Halliley JL, Chaves FA, et al. Broad hemagglutinin-specific memory B cell expansion by seasonal influenza virus infection reflects early-life imprinting and adaptation to the infecting virus. J Virol. 2019;93:e00169-19. 